\title{
Constructal design applied to the elastic buckling of thin plates with holes
}

Research Article

Luiz A. O. Rocha ${ }^{1}$, Liércio A. Isoldi ${ }^{2}$, Mauro de Vasconcellos Real ${ }^{2}$, Elizaldo Domingues dos Santos ${ }^{2}$, Anderson L. G. Correia ${ }^{2}$, Giulio Lorenzini ${ }^{3 *}$, Cesare Biserni ${ }^{4}$

1 Department of Mechanical Engineering, Universidade Federal do Rio Grande do Sul, Rua Sarmento Leite, 425, Porto Alegre, RS 90.050-170, Brazil.

2 School of Engineering, Universidade Federal do Rio Grande, Cx.P. 474, Rio Grande, RS 96201-900, Brazil

3 Department of Industrial Engineering, University of Parma, Parco Area delle Scienze 181/A, Parma 43124, Italy

4 Department of Industrial Engineering, University of Bologna, Viale Risorgimento 2, Bologna 40136, Italy

Received 19 April 2013; accepted 10 June 2013

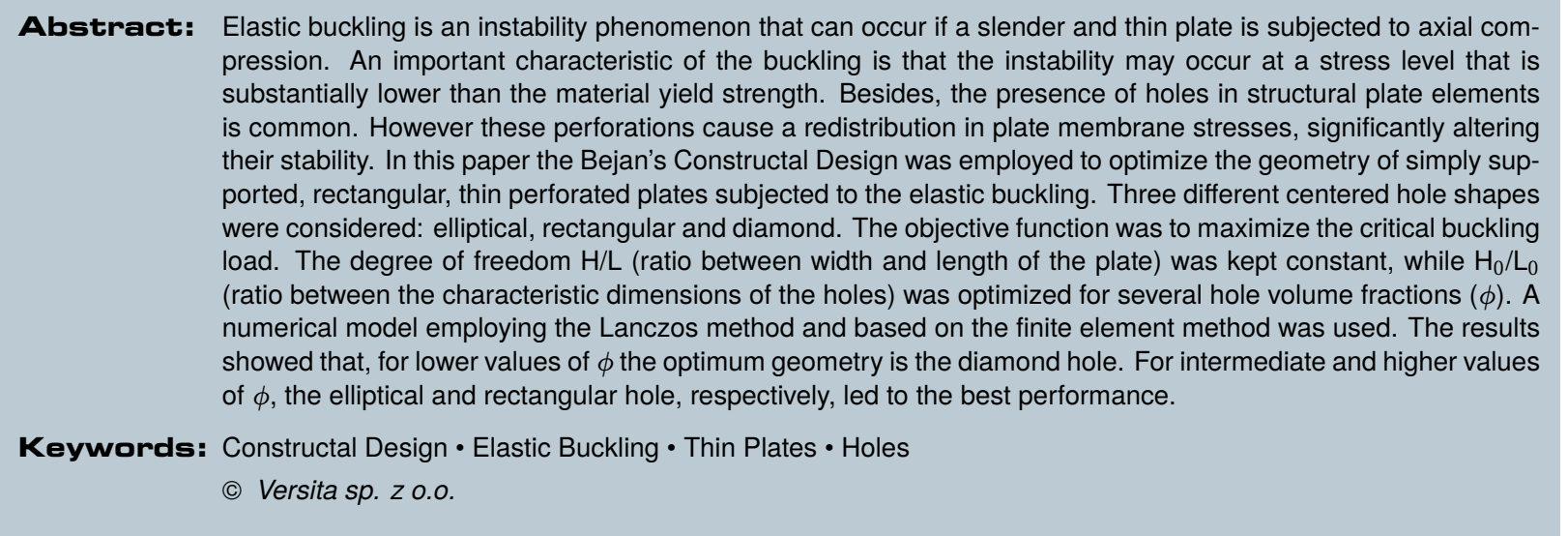

\section{Introduction}

Slender structural elements subjected to axial compressive loads can fail suddenly due to a phenomenon known as buckling instability [1]. The plates are thin structural elements commonly used in buildings, bridges, ships, planes and automobiles. In many cases, it is almost in- 
evitable to have holes in these plate elements for inspection, maintenance, service, and weight reduction purposes. The presence of holes causes a redistribution of the membrane stresses accompanied by a change in the mechanical behavior of the plate [2]. When these structural components are subjected to axial compression the buckling phenomenon can occur. At a certain given critical load the plate will very sudden present large deflections in the out-of-plane transverse direction [3]. An important characteristic of the buckling phenomenon is that this instability may occur at a stress level that is substantially lower than the material yield strength. The buckling behavior of perforated plates has been the object of a large number of researches in the last decade. The analyses methods adopted in the published articles can be divided into two categories, i.e., linear elastic buckling and nonlinear elasto-plastic buckling. Among the elastic buckling studies category, El-Sawy and Nazmy [4] using a numerical approach investigated the effect of aspect ratio on the elastic buckling critical loads of uniaxially loaded rectangular plates with eccentric circular and rectangular (with rounded corners) holes. El-Sawy and Martini [5] used the finite element method to determine the elastic buckling stresses of biaxially loaded perforated rectangular plates with longitudinal axis located circular holes. Alternatively, Moen and Schafer [6] developed, validated and summarized analytical expressions for estimating the influence of single or multiple holes on the elastic buckling critical stress of plates in bending or compression. In the group of studies dedicated to the problem of elasto-plastic buckling, El-Sawy et al. [7] investigated the elasto-plastic buckling of uniaxially loaded square and rectangular plates with circular cutouts by the use of the finite element method, including some recommendations about hole size and location for the perforated plates of different aspect ratios and slenderness ratios. Afterwards, Paik [8-10] studied the ultimate strength characteristics of perforated plates under edge shear loading, axial compressive loading and the combined biaxial compression and edge shear loads, and proposed closed-form empirical formulae for predicting the ultimate strength of perforated plates based on the regression analysis of the nonlinear finite element analyses results. Maiorana et al. $[11,12]$ focused on the linear and nonlinear finite element analyses of perforated plates subjected to localized symmetrical load. In summary, most of the previous researches focused on the changes in buckling behavior of perforated plates due to the presence of cutouts. The optimal hole shape and hole size to the plate buckling performance has not yet been systematically investigated. This task can be appropriately performed by relying in Constructal theory and has a practical relevance as in the self-weight reduc- tion of structures. Constructal theory is the view that the generation of flow configurations is a physics phenomenon that can be based on a physics principle (the Constructal law). The Constructal law states that for a finite-size flow system to persist in time (to live), its configuration must evolve in such a way that it provides easier access to the currents that flow through it [12-16]. Constructal design is the method based on constructal law to discover the configurations that maximize the access of the flow currents. The method applies the objective and constraints principle in such a way that the best architecture can emerge deterministically. The applicability of this method to engineered flow systems has been widely discussed in recent literature, e.g. in designing cavities and assembly of fins $[17,18]$. Lorente et al. [19] have shown that Constructal theory can also be applied to mechanical strength. Therefore, the main objective of this article is to show the application of the Constructal Design to the determination of the optimal hole shape and size to the elastic buckling performance of perforated plates (with elliptical, rectangular and diamond holes) under uniaxial compression loading.

\section{Method of analysis}

Initially the solid (non-perforated) plate was studied analytically and numerically. The results obtained by these two approaches were compared, serving as the first validation of the computational modeling. Besides, the analytical critical buckling load for the plate without hole was adopted as a reference parameter throughout the work. After that, a second validation of the numerical model was performed by comparing the results for the critical buckling load of perforated plates with numerical results obtained by other authors. Finally, the geometrical optimization process for plates with centered holes based on Bejan's Constructal design [13] was developed.

\subsection{Buckling of plates}

In many design situations, thin plates are subjected to uniaxial compressive loads. The slenderness of these components causes them to be susceptible to instabilities such as buckling. An example of a rectangular thin plate of length $L$, width $H$, thickness $t$ and subject to axial compressive loads $P$ can be seen in Figure 1 .

While these in-plane forces $P$ are kept sufficiently small, the equilibrium remains stable and the resulting deformations are characterized by the absence of lateral (out-ofplane) displacements. If the magnitude of these in-plane forces is increased, at certain load intensity, the plate 


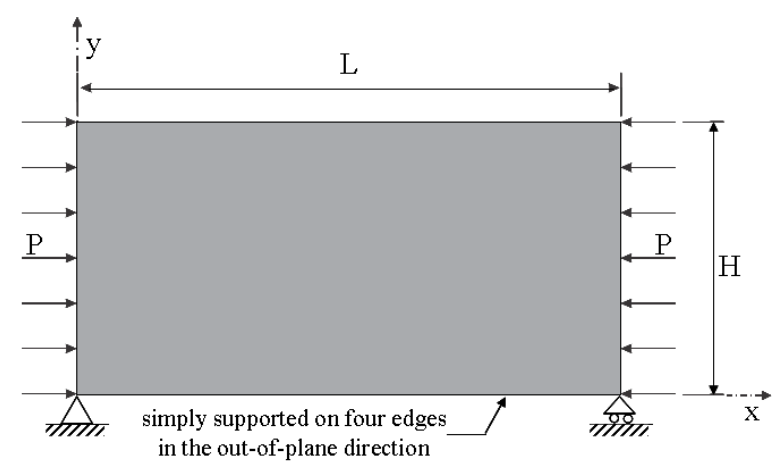

Figure 1. Rectangular solid plate subject to uniaxial compressive load.

will suddenly present large deflections in the out-of-plane transverse direction. In this condition, the originally stable equilibrium becomes unstable and the plate is said to have buckled. The load producing this condition is called the critical load. The importance of the critical load is the initiation of a deflection pattern that, if the load is further increased, rapidly leads to very large lateral deflections and eventually to complete failure of the plate. For the case of a rectangular thin plate the value of the critical load $P_{c r}$ can be determined analytically, as it is shown in Timoshenko and Gere [20] and in Wang et al. [21], in the form of the following equation

$$
P_{c r}=\left(\kappa \pi^{2} E t^{3}\right) /\left[12 H^{2}\left(1-v^{2}\right)\right]
$$

where $\kappa$ is a mathematical constant, $E$ and $v$ are the Young's modulus and Poisson's ratio of the material, respectively, and $k$ is the buckling coefficient given by:

$$
k=[m(H / L)+(1 / m)(L / H)]^{2}
$$

where $m$ is the number of half waves that occur on plate buckling in the longitudinal direction, defining the buckling mode of the plate. The analytical solution for the critical buckling stress is obtained by dividing the critical load $P_{c r}$ by the plate thickness $t$, resulting,

$$
\sigma_{c r}=k \pi^{2} E /\left[12\left(1-v^{2}\right)(H / t)^{2}\right]
$$

where the ratio $H / t$ is called the plate slenderness. The materials properties and dimensions of the plates analyzed are shown in Table 2.1.

By applying Equation (2), using the data from Table 1 and assuming that two half waves are generated in the longitudinal direction of the plate, one obtains the buckling coefficient $k=4.0$. Now using Equation (1), the analytical value for the critical buckling load in this example is $P_{c r}=759.20 \mathrm{kN} / \mathrm{m}$. The numerical model was developed through the finite element software ANSYS ${ }^{\circledR}$. In all
Table 1. Plates materials properties and dimensions

\begin{tabular}{cc}
\hline Parameter & Value \\
\hline$E$ & $210.0 \mathrm{GPa}$ \\
\hline$v$ & 0.3 \\
\hline$H$ & $1.0 \mathrm{~m}$ \\
\hline$L$ & $2.0 \mathrm{~m}$ \\
\hline$t$ & $10.0 \mathrm{~mm}$ \\
\hline
\end{tabular}

simulations the SHELL93 element, with eight nodes and reduced integration, was used. This element has six degrees of freedom at each node: three translations $(u, v, w)$ and three rotations $\left(\Theta_{x}, \Theta_{y}, \Theta_{z}\right)$ [22]. For the numerical study of the buckling phenomenon it was adopted an elastic eigenvalue analysis. The finite elements equilibrium equations for this type of analysis involve the solution of a homogeneous algebraic equation system whose lowest eigenvalue corresponds to the critical buckling load and the associated eigenvector represents the first buckling mode of the plate [23]. The formulation used in the analysis includes both the linear and non-linear strain components. Thus, the total stiffness matrix $[K]$ is obtained by the sum of the conventional stiffness matrix for small deformations, $\left[K_{E}\right]$, with another matrix $\left[K_{G}\right]$, called geometrical stiffness matrix. The matrix $\left[K_{G}\right]$ depends not only on the geometry but also on the internal forces existing at the beginning of loading, $\left\{\mathrm{P}_{0}\right\}$. Then, the total stiffness matrix for a plate at the load level $\left\{\mathrm{P}_{0}\right\}$ can be written as [24]:

$$
[K]=\left[K_{E}\right]+\left[K_{G}\right]
$$

When the load reaches a level $\{P\}=\lambda\{P 0\}$, where $\lambda$ is a scalar, the stiffness matrix can be defined as:

$$
[K]=\left[K_{E}\right]+\lambda\left[K_{G}\right]
$$

The governing equilibrium equations for the plate can be written as:

$$
\left[\left[K_{E}\right]+\lambda\left[K_{G}\right]\right]\{U\}=\lambda\left\{P_{0}\right\}
$$

where $\{U\}$ is the total displacement vector, which may therefore be given by:

$$
\{U\}=\left[\left[K_{E}\right]+\lambda\left[K_{G}\right]\right]^{-1} \lambda\left\{P_{0}\right\}
$$

At buckling load, the plate presents a large increase in the displacements without a load increase. By mathematical definition it is possible to determine the inverse matrix as the adjoint matrix divided by the determinant of the coefficients, then the displacements $\{U\}$ tend to infinity when: 


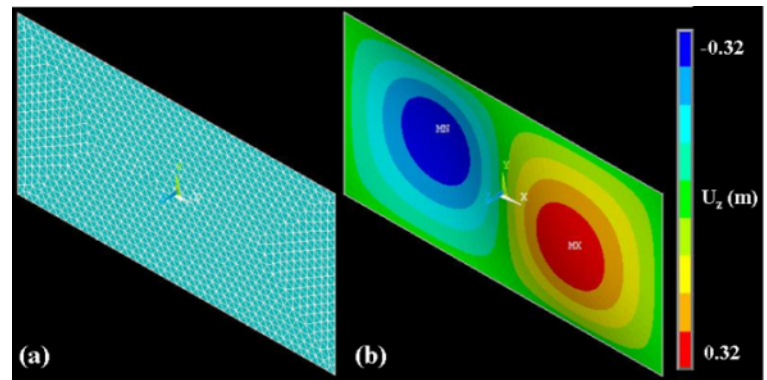

Figure 2. Non-perforated plate: (a) finite elements mesh; (b) First buckling mode shape.

$$
\operatorname{det}\left[\left[K_{E}\right]+\lambda\left[K_{G}\right]\right]=0
$$

Equation (8) represents an eigenvalue/eigenvector mathematical problem. The solution of this problem produces the smallest eigenvalue, $\lambda_{1}$, which corresponds to the critical load $\left\{P_{c r}\right\}=\lambda_{1}\left\{P_{0}\right\}$ at which the buckling phenomenon occurs. Furthermore, the displacement vector associated $\{U\}$ (eigenvector) defines the buckling mode shape. In the program ANSYS ${ }^{\circledR}$, the eigenvalue/eigenvector problem is solved by using the numerical method of Lanczos [22].

\subsubsection{Buckling of non-perforated plates}

To verify the computational model, the critical load of a non-perforated plate was numerically evaluated and the result was compared with the analytical solution given by Equation (1). The plate was discretized and grid independence was reached by adopting a triangular element with sides of size $50.00 \mathrm{~mm}(H / 20)$, generating a mesh with 1,814 finite elements (Figure 2 (a)). The numerical result for the critical buckling load was $755.30 \mathrm{kN} / \mathrm{m}$. This means a difference of $-0.51 \%$ between numerical and analytical solutions. Figure 2 (b) shows the buckling mode of non-perforated plate.

\subsubsection{Buckling of perforated plates}

Here, the computational model previously presented was employed to analyze the buckling behavior of thin perforated plates. The same plate used in the first verification was studied, however a centered circular hole were considered. In Table 2.1.2 the results for the critical buckling load were compared with those obtained by the numerical study developed by El-Sawy and Nazmy [4].

Again an excellent agreement was obtained, being $-0.53 \%$ the maximal difference encountered.
Table 2. Comparison of critical buckling load for plate with centered circular hole

\begin{tabular}{|c|c|c|c|}
\hline $\begin{array}{c}\text { Hole } \\
\text { diamenter }(\mathrm{m})\end{array}$ & $\begin{array}{c}P_{c r}(\mathrm{kN} / \mathrm{m}) \\
\text { El-Sawy and } \\
\text { Nazmy [4] }\end{array}$ & $\begin{array}{c}P_{c r}(\mathrm{kN} / \mathrm{m}) \\
\text { Present work }\end{array}$ & $\begin{array}{c}\text { Difference } \\
(\%)\end{array}$ \\
\hline 0.10 & 766,19 & 763.56 & -0.34 \\
\hline 0.20 & 789.36 & 786.50 & -0.36 \\
\hline 0.30 & 825.08 & 820.87 & -0.51 \\
\hline 0.40 & 849.26 & 847.78 & -0.17 \\
\hline 0.50 & 901.54 & 898.79 & -0.31 \\
\hline 0.60 & 986.46 & 981.22 & -0.53 \\
\hline
\end{tabular}

\subsection{Constructal design applied to the elastic buckling of plates}

Constructal design was employed in this section to study thin plates with a centered perforation. Three different types of holes were considered: elliptical, rectangular and diamond. The objective is to maximize the critical buckling load. The volume of the plate was constant and given by

$$
V=H L t
$$

The volume of the hole $\left(V_{0}\right)$ was also kept constant, but this constraint was replaced by the hole volume fraction $(\phi)$, which is defined by the ratio between the volume of the hole and the volume of the plate. The ratio between the width of plate and its length $\mathrm{H} / \mathrm{L}$ was also kept constant, but the ratio $H_{0} / L_{0}$, the characteristic dimensions of the hole, was allowed to vary. Therefore, the variation of the size of the holes is governed by the parameter hole volume fraction $(\phi)$. So to the plate with a centered elliptical hole (Figure 3), the volume fraction is defined by:

$$
\phi=\frac{V_{0}}{V}=\frac{\pi H_{0} L_{0} t / 4}{H L t}=\frac{\pi H_{0} L_{0}}{4 H L}
$$

where $\pi$ is the mathematical constant; $H_{0}$ and $L_{0}$ are the characteristic dimensions of hole in $y$ and $x$ directions, respectively; $H$ is the plate width, $L$ is the plate length and $t$ is the plate thickness.

When the thin plate has a hole with a rectangular shape (Figure 4) the hole volume fraction is given by:

$$
\phi=\frac{V_{0}}{V}=\frac{H_{0} L_{0} t}{H L t}=\frac{h_{0} L_{0}}{H L}
$$

And for the plate with centered diamond hole (Figure 5) the hole volume fraction is obtained by:

$$
\phi=\frac{V_{0}}{V}=\frac{\left(H_{0} L_{0} t\right) / 2}{H L t}=\frac{h_{0} L_{0}}{H L}
$$




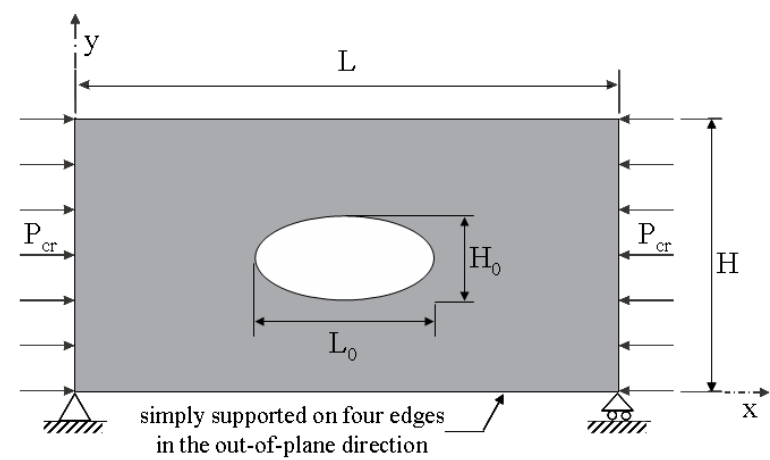

Figure 3. Plate with centered elliptical hole.

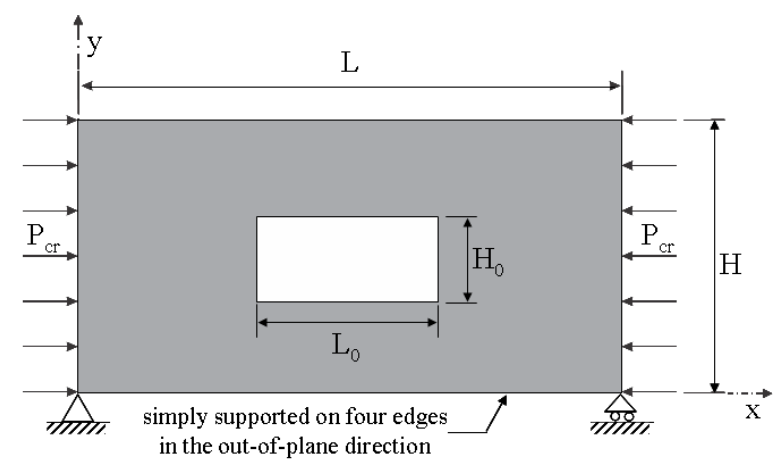

Figure 4. Plate with centered rectangular hole.

The objective in all analyses was to determine the optimal hole geometry $\left(H_{0} / L_{0}\right)$ that is characterized by the maximization of critical buckling load for the perforated plate. For this, based on Constructal design, the variables of the problem were considered dimensionless:

$$
\widetilde{x}, \widetilde{y}, \widetilde{t}, \widetilde{H}, \widetilde{L}, \widetilde{H_{0}}, \widetilde{L_{0}}=\frac{x, y, t, H, L, H_{0}, L_{0}}{A^{1 / 2}}
$$

being $A$ the area of plate without hole defined as:

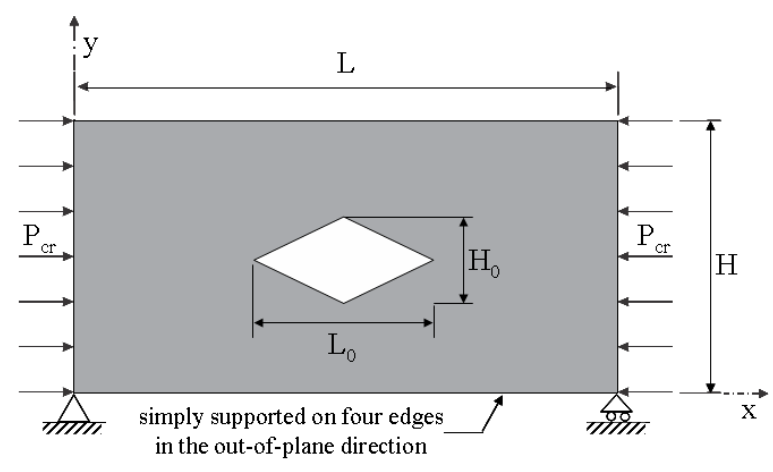

Figure 5. Plate with centered diamond hole.

$$
A=H L
$$

and its dimensionless definition is given by

$$
1=\tilde{H} \widetilde{L}
$$

It is worthy to mention that, the diversity of phenomena addressed with several ad hoc statements and optimization methods (e.g., minimum entropy generation, maximum entropy production, minimum and maximum flow resistance and others) are manifestations of the single natural tendency that has been expressed by the Constructal Law [13-16]. Moreover, the applicability of Constructal Design in association with numerical methods, as the Finite Element Method (FEM), has been confronted with other optimization methods [13-16]. Examples of this applicability for several engineering problems have been presented in recent literature $[17,18,25]$.

\section{Results and discussion}

The investigation about the critical buckling load of perforated plates was developed considering different values for the hole volume fraction, $\phi$ for each hole shape. So, a variation of the degree of freedom $H_{0} / L_{0}$ was performed for each case. The other degree of freedom, $H / L$, was kept equal to 0.50 for all numerical simulations. Besides, these values of critical buckling load numerically obtained were normalized by the analytical result for the critical buckling load of a non-perforated plate (Equation (1)), i.e.,

$$
P_{c r, d i m}=\frac{P_{c r, d i m}}{P_{c r}}
$$

Figure 6 presents the behavior of dimensionless critical buckling load as function of the degree of freedom HO/LO of the plate with centered elliptical hole. It is possible to note in Figure 6 that for each hole volume fraction there is a maximum value of critical buckling load that is placed in an intermediate range of the ratio $H_{0} / L_{0}$. Another important observation among the results presented in Figure 6 is concerned with the increasing of the maximum critical buckling load with the increasing of the hole volume fraction. This trend is related with the degree of freedom $H / L$ equal to 0.5 , i.e., other values of $H / L$ may conduct to different behaviors.

The buckled shapes for a plate with a centered elliptical hole are shown in Figure 7. These shapes correspond to three values of $H_{0} / L_{0}: 0.25$ (Figure 7(a)), 0.90 (Figure 7(b)) and 1.00 (Figure 7(c)), which represents the lower extreme, the optimal and the upper extreme ratios of $H_{0} / L_{0}$, respectively. This plate has a hole volume fraction of 0.30 . 


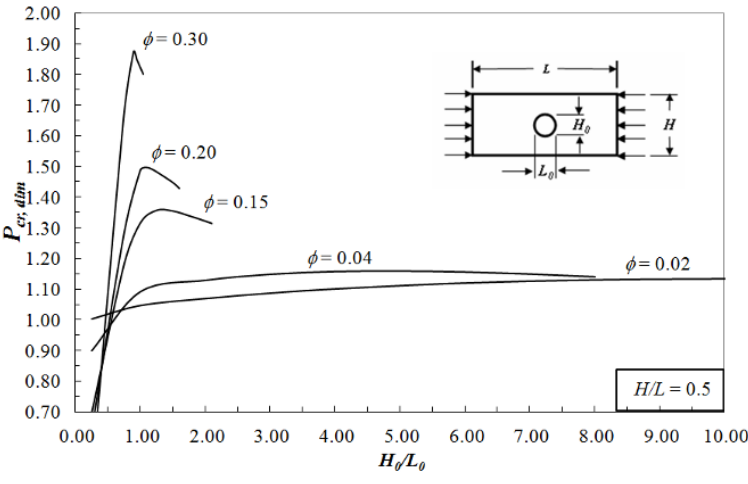

Figure 6. Plate with centered elliptical hole: optimization of the critical buckling load.

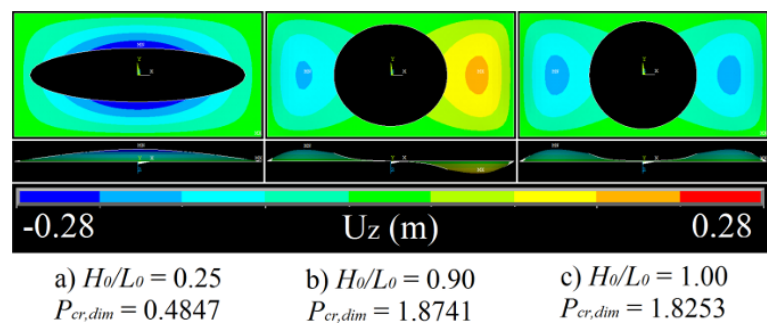

Figure 7. Buckled shape of elliptical perforated plate with hole volume fraction of 0.30 .

The plate behavior of Figure 7(a) indicates the formation of only one half-wave in buckling, while the topologies in Figure 7(b) and Figure 7(c) show the formation of two halfwaves. This trend is confirmed if the values of dimensionless critical buckling load are compared: it is necessary a higher intensity of load to produce two half-waves in the plate than to produce one half-wave. It is also observed that the best shape, Figure 7(b), improves the dimensionless buckling load in $286.6 \%$ and $2.7 \%$ in comparison with the lower and upper extremes, Figure 7(a) and Figure 7(c). The results for the plate with rectangular centered hole are showed in Figure 8. The evolution of dimensionless critical buckling load is plotted as a function of degree of freedom $H_{0} / L_{0}$ for the same values of hole volume fraction already mentioned. An analogous behavior in comparison with that of Figure 6 is observed, i.e., when the value of hole volume fraction increases there is an increase in maximum $P_{c r, d i m}$.

Considering $\phi$ of 0.40 and HO/LO of $0.25,0.75$ and 1.00, the buckled shapes for rectangular perforated plate are presented in Figure 9(a), Figure 9(b) and Figure 9(c), respectively. The maximum critical buckling load is obtained for $H_{0} / L_{0}$ 0.75, Figure 9 (b).

Finally, the plate with a centered diamond hole was analyzed, and the results for the dimensionless critical buck-

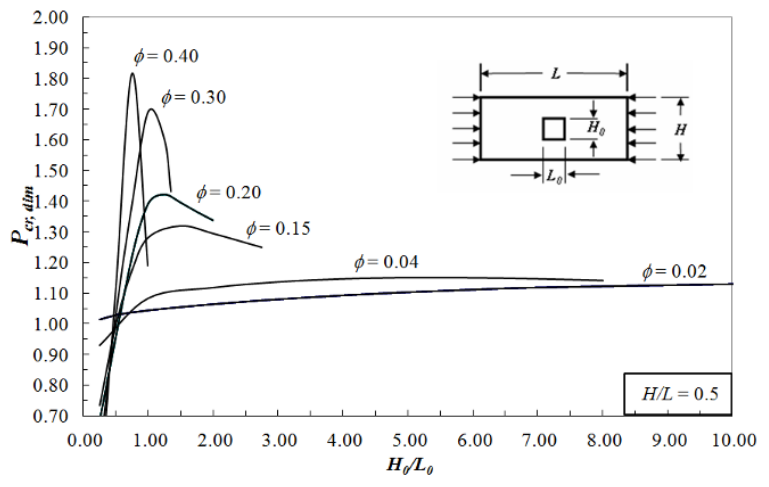

Figure 8. Plate with centered rectangular hole: optimization of the critical buckling load.

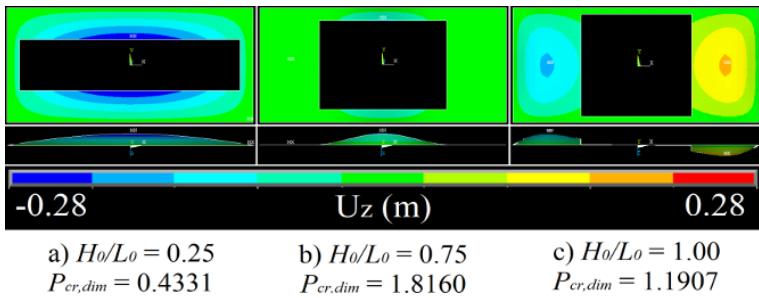

Figure 9. Buckled shape of rectangular perforated plate with hole volume fraction of 0.40 .

ling load by varying the ratio $H_{0} / L_{0}$ are presented in Figure 10.

Comparing the behavior illustrated in Figure 10 with those presented in Figure 6 and in Figure 8 , it is possible to identify the same trend, i.e., only one value for the maximal critical buckling load is obtained as a function of the degree of freedom $H_{0} / L_{0}$. Figure 11 shows the topologies of the buckling mode shapes, for the diamond perforated plate, with hole volume fraction of $\phi=0.20$ and the fol-

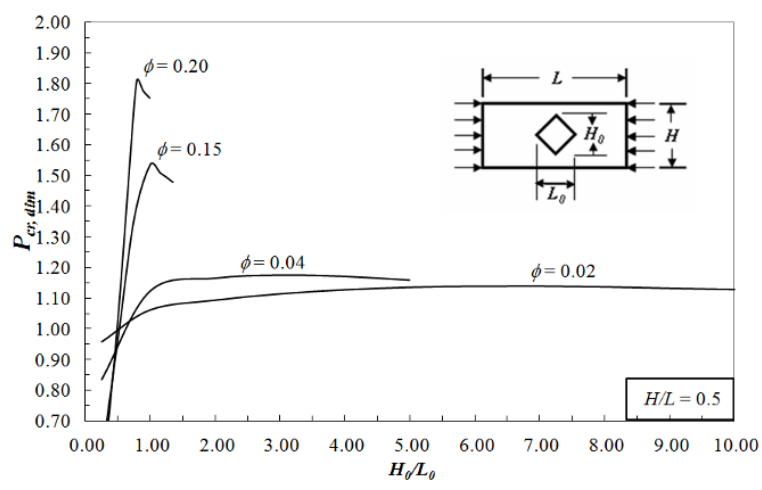

Figure 10. Plate with centered diamond hole: optimization of the critical buckling load. 


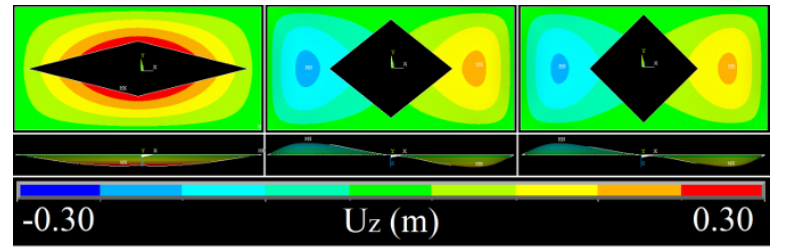

a) $H_{0} / L_{0}=0.25$

$P_{\text {cr, dim }}=0.4771$

b) $\mathrm{H}_{0} / \mathrm{L}_{0}=0.80$

c) $H_{0} / L_{0}=1.00$

$P_{\text {cr, dim }}=1.8112$

$P_{c r, \operatorname{dim}}=1.7519$

Figure 11. Buckled shape of diamond perforated plate with hole volume fraction of 0.20 .

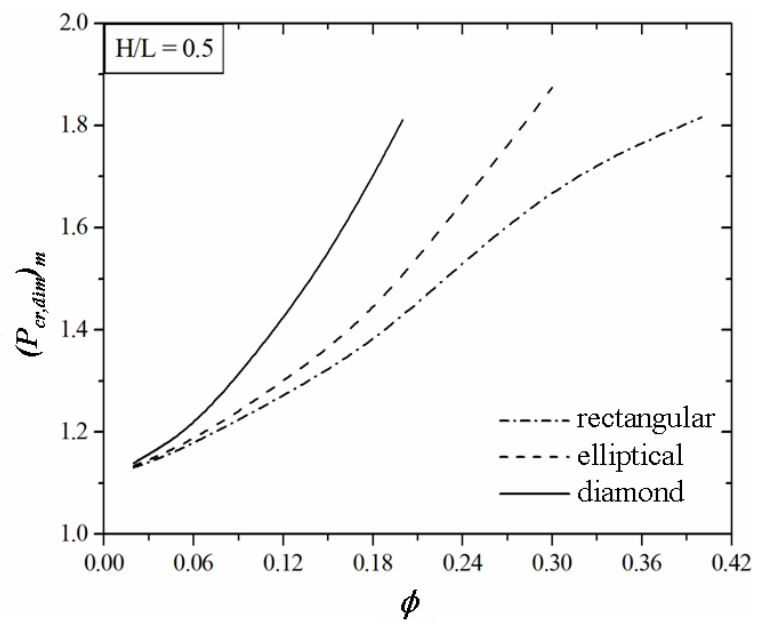

Figure 12. The once maximized dimensionless critical buckling load $\left(P_{c r, d i m}\right) \mathrm{m}$ as a function of the hole volume fraction $\phi$ for all hole shapes.

lowing ratios of $H_{0} / L_{0}: 0.25$ (Figure 11(a)), 0.80 (Figure $11(\mathrm{~b}))$ and 1.00 (Figure 11(c)).

It is noted that for the small value of $H_{0} / L_{0}$, which corresponds to a higher intrusion of the hole in longitudinal direction, only one half-wave is generated, as already observed in other hole shapes (see Figure 7(a) and Figure 9(a)). The other two topologies are very similar (Figure 11(b) and 11(c)). Each one is formed by two half-waves and having displacements distributed more uniformly than that reached for Figure 11(a). Therefore, it is presented an analogous behavior to that performed for the elliptical hole. Beyond the already mentioned hole volume fractions, other values of $\phi$ were numerically investigated. Figure 12 exhibits the effect of the hole volume fraction over the once maximized dimensionless buckling load for the three hole shapes studied. It is emphasized that, it is not possible to obtain geometries for the diamond and elliptical holes at values of $\phi>0.2$ and $\phi>0.3$, respectively.

Figure 12 indicates that, for $\phi 0.20$, higher values of critical buckling loads are obtained for plates with centered

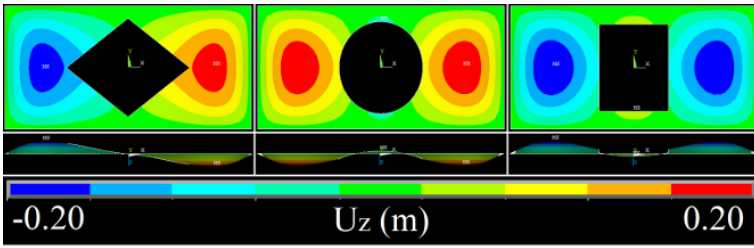

$\begin{array}{lll}\text { a) } H_{0} / L_{0}=0.80 & \text { b) } H_{0} / L_{0}=1.10 & \text { c) } H_{0} / L_{0}=1.25 \\ P_{c r, d i m}=1.8112 & P_{c r, d i m}=1.4967 & P_{c r, d i m}=1.4216\end{array}$

Figure 13. Comparison of the optimized buckled shape of plate with rectangular, diamond and elliptical hole for the hole volume fraction of 0.20 .

diamond hole, i.e., in the range where the comparison between the three geometries is allowed, the diamond hole leads to the best performance. For $\phi=0.20$, the highest dimensionless buckling load is $P_{c r, d i m}=1.8112$, which is approximately $17.4 \%$ and $21.5 \%$ better than the best elliptical and rectangular shapes, respectively. For the range $0.20<\phi \leq 0.30$, the elliptical shape is better than the rectangular one, reaching a maximum $P_{c r, d i m}=1.8741$, which is approximately $10 \%$ higher than the $P c r$,dim found for the best rectangular shape. For $0.3<\phi \leq 0.4$, it is only possible to intrude rectangular holes into the plate. In spite of this fact, the maximum dimensionless buckling load reached for the rectangular hole at $\phi=0.4$ is $P_{c r, \text { dim }}=1.8160$, which is $3.0 \%$ lower than the optimal elliptical shape for $\phi=0.3$. The results make evident the importance of applying the Constructal Design for the geometric optimization of engineering structures. Supposing a practical situation of structural engineering design, where it would be necessary or desirable the employment of a simply supported perforated plate subject to uniaxial compression load with a hole volume restriction of $\phi=$ 0.2 . Figure 13 shows that, on the contrary to intuitively expected, the optimal shape is obtained with the diamond hole. It is also observed that, the optimal shape is that one that leads to the best distribution of imperfections (displacement, stress). In other words, the same principle employed for the optimization of fluid mechanics and heat transfer problems, and also employed for deterministically explain the nature shapes, is also the responsible for the geometry in problems of mechanic of materials [19].

Finally, in Figure 14 it is depicted the once optimized ratio of $H_{0} / L_{0},\left(H_{0} / L_{0}\right)_{0}$, as a function of for the three hole shapes: elliptical, rectangular and diamond One can observe that the same trend is exhibited for all kinds of hole: the once optimized rate $\left(H_{0} / L_{0}\right)$ o decreases with the increase of the hole volume fraction $\phi$. For the same value of $\phi$, the results indicate that the best performance is reached for the geometry with the lowest once optimized ratio of $\left(H_{0} / L_{0}\right)_{0}$.

Here it is important to note that the method used in the 


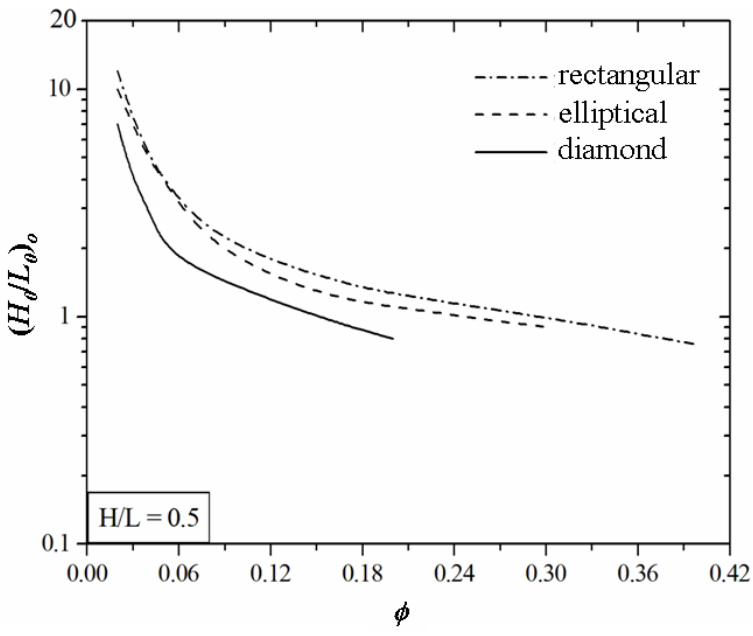

Figure 14. The once optimized rate of $\left(H_{0} / L_{0}\right)$ o as a function of the hole volume fraction $\phi$ for all hole shapes.

determination of the plate buckling load assumes a linear elastic material behavior. Thus, the results here presented are rigorously valid only if the calculated critical buckling load does not cause normal stresses above the material's yield strength, when it is applied to the plate. If the yielding stress limit is surpassed, a nonlinear finite element analysis should be performed, including geometrical and material nonlinearities [25]. An application of these results could be a case when it is necessary to reduce the weight of plates that compose the web of bridge girders or the web of offshore structures columns. Here the purpose is to withdraw a certain amount of plate's material (defined by the $\phi$ parameter) without affecting the plate load bearing capacity. If a hole volume fraction of $20 \%$ is considered, the recommended hole shape would be the diamond as it is shown in Fig. 13. In this case even a higher elastic buckling critical load could be achieved.

\section{Conclusion}

The importance of thin perforated plates as structural members is evident in many engineering applications such as buildings, bridges, ships, planes and automobiles. Therefore, this work was developed aiming to obtain the best geometry that provides the maximum critical elastic buckling load. A numerical approach, based on the $\mathrm{Fi}-$ nite Element Method (FEM), was adopted to apply the concepts of Constructal Design, defining the optimal geometry, i.e., the geometry that supports the highest critical buckling load. Three different configurations for the centered hole in a perforated thin plate were considered: elliptical, rectangular and diamond. The influence of the hole shape variation and the hole size variation was investigated, adopting a constant ratio between width and length of the plate The results indicated that the plate with diamond hole is the best geometry until a volume ratio $\phi$ of 0.20 is reached, i.e., higher values of the critical buckling loads are obtained for plates with centered diamond hole for $\phi 0.20$. In this range the maximum dimensionless critical buckling load $P_{c r, d i m}$ encountered was approximately 1.80. However, for hole volume fractions between 0.20 and 0.30 the hole shape that generates larger critical buckling loads is the elliptical, reaching a maximum $P_{c r, d i m}$ of approximately 1.90 when $\phi=0.30$. For the cases where volume fractions larger than 0.30 are required, it was indicated the rectangular shape for the central perforation in the plate, reaching a maximum $P_{c r \text {,dim }}$ of approximately 1.80 for $\phi=0.40$. One can note that the optimized geometries were defined in agreement with the Constructal principle of minimization of distribution of imperfections, showing that the Constructal Design can be used to obtain the optimal shapes in mechanic of materials problems with the same efficiency that is used in problems of fluid mechanics and heat transfer. It is interesting to note that thin plates present a different behavior from columns with respect to buckling. For a slender column the buckling load almost coincides with its failure load. On the other hand, thin plates can support a considerable load amount after buckling due to stress redistribution and elasto-plastic material behavior. However, in the present work the analyses were limited to the elastic buckling of plate. So a natural sequence of this work is the investigation of the structural behavior of perforated thin plates under uniaxial compression considering both geometrical and material nonlinearities.

\section{Acknowledgements}

The authors thank CAPES (Brazilian Coordination for Improvement of Higher Education Personnel) and $\mathrm{CNPq}$ (Brazilian National Council for Scientific and Technological Development) for the financial support.

\section{Conflict of interest}

The authors do not have a direct financial relation with the commercial identity mentioned in this paper, avoiding any conflict of interest. 


\section{References}

[1] Megson T. H. G., Structural and Stress Analysis, Ed. Elsevier Butterworth-Heinemann, 2005.

[2] Cheng B., Zhao J., Strengthening of perforated plates under uniaxial compression: Buckling analysis, ThinWalled Structures, vol. 48, no. 12, 905-914, 2010

[3] Åkesson B., Plate buckling in bridges and other structures, Taylor \& Francis, 2007.

[4] El-Sawy K.M., Nazmy A.S., Effect of aspect ratio on the elastic buckling of uniaxially loaded plates with eccentric holes, Thin-Walled Structures, vol. 39, no. 12, 983-998, 2001

[5] El-Sawy K. M., Martini M. I., Elastic stability of biaxially loaded rectangular plates with a single circular hole, Thin-Walled Structures, vol. 45, no. 1, 12233, 2007

[6] Moen D., Schafer B. W., Elastic buckling of thin plates with holes in compression or bending, ThinWalled Structures, vol. 47, no. 12, 1597-1607, 2009

[7] El-Sawy K. M., Nazmy A. S., Martini M. I., Elastoplastic buckling of perforated plates under uniaxial compression, Thin-Walled Structures, vol. 42, no. 8, 1083-1101, 2004

[8] Paik J.K., Ultimate strength of perforated steel plates under edge shear loading, Thin-Walled Structures, vol. 45, no. 3, 301-306, 2007

[9] Paik J.K., Ultimate strength of perforated steel plates under axial compressive loading along short edges, Ships and Offshore Structures, vol. 2, no. 4, 355-360, 2007

[10] Paik J.K., Ultimate strength of perforated steel plates under combined biaxial compression and edge shear loads, Thin-Walled Structures, vol. 46, no. 2, 207-213, 2008

[11] Maiorana E., Pellegrino C., Modena C., "Linear buckling analysis of perforated plates subjected to localized symmetrical load", Engineering Structures, vol. 30, 3151-3158, 2008

[12] Maiorana E., Pellegrino C., Modena C., Non-linear analysis of perforated steel plates subjected to local- ized symmetrical load, Journal of Constructional Steel Research, vol. 65, 959-964, 2009

[13] Bejan A., Shape and Structure, from Engineering to Nature, Cambridge University Press, Cambridge, UK, 2000

[14] Bejan A., Lorente S., Design with Constructal Theory, Wiley, Hoboken, 2008

[15] Bejan A., Advanced Engineering Thermodynamics, 2nd ed., Willey, New York, 1997

[16] Bejan A., Zane P., Design in Nature, 1st ed., Doubleday, 2012

[17] Biserni C., Rocha L. A. O., Stanescu G., Lorenzini E., Constructal H-shaped cavities according to Bejan's theory, International Journal of Heat Mass Transfer, vol. 50, 2132-2138, 2007

[18] Lorenzini G., Correa R.L., Dos Santos E.D., Rocha L.A.O., Constructal Design of Complex Assembly of Fins, Journal of Heat Transfer-Transactions of the ASME, vol. 133, no. 8, 081902 (7 pages), 2011

[19] Lorente S., Lee J., Bejan A., The "flow of stresses" concept: the analogy between mechanical strength and heat convection, International Journal of Heat Mass Transfer, vol. 53, 2963-2968, 2010

[20] Timoshenko S. P., Gere J. M., Theory of Elastic Stability, 2nd ed., McGraw-Hill Book Company, New York, 1961

[21] Wang C. M., Wang C.Y., Reddy J.N., Exact solutions for buckling of structural members, CRC Press, 2005

[22] ANSYS, "User's Manual (version 10.0)", Houston, Swanson Analysis System Inc, 2005

[23] Madenci E., Guven I., The Finite Element Method and Applications in Engineering Using ANSYSő, Ed. Springer, 2006

[24] Przemieniecki J. S., Theory of Matrix Structural Analysis, Ed. Dover Publications, 1985

[25] Rocha L.A.O., Real M.V., Correia A.L.G., Vaz J., et al., Geometric Optimization Based on the Constructal Design of Perforated Thin Plates Subject to Buckling, Computational Thermal Sciences, vol. 4, no. 2, 119-129, 2012 